Analysis Name: $30 \mu g$ Exenatide_vs_control

Analysis Creation Date: 2013-12-06

Build version: 261899

Content version: 17199142 (Release Date: 2013-09-17)

\title{
Analysis settings
}

\section{View}

Reference set: Mouse Genome 430 2.0 Array

Relationship to include: Direct and Indirect

Includes Endogenous Chemicals

Optional Analyses: My Pathways My List

Filter Summary:

Consider only relationships where

(confidence $=$ Experimentally Observed) AND

(data sources = An Open Access Database of Genome-wide Association Results OR BIND OR BIOGRID OR Catalogue Of Somatic Mutations In Cancer (COSMIC) OR Chemical Carcinogenesis Research Information System (CCRIS) OR ClinicalTrials.gov OR ClinVar OR Cognia OR DIP OR DrugBank OR Gene Ontology (GO) OR GVK Biosciences OR Hazardous Substances Data Bank (HSDB) OR HumanCyc OR Ingenuity Expert Findings OR Ingenuity ExpertAssist Findings OR INTACT OR Interactome studies OR MINT OR MIPS OR miRBase OR miRecords OR Mouse Genome Database (MGD) OR Obesity Gene Map Database OR Online Mendelian Inheritance in Man (OMIM) OR TarBase OR TargetScan Human)

Cutoff:

Fold Change $=1.300$

$\mathrm{p}$-value $=5.00 \mathrm{E}-02$ 


\section{Top Networks}

ID Associated Network Functions

Embryonic Development, Organismal Development, Tissue Development

Embryonic Development, Tissue Development, Tissue Morphology

40

Post-Translational Modification, Protein Degradation, Protein Synthesis

39

Hereditary Disorder, Metabolic Disease, Post-Translational Modification

37

5 Molecular Transport, Dermatological Diseases and Conditions, Organ Morphology 
Top Diseases and Bio Functions

\section{Diseases and Disorders}

Name

p-value

\#

Endocrine System Disorders

6.86E-06 - 1.88E-02 57

Hematological Disease

$6.86 \mathrm{E}-06-2.68 \mathrm{E}-0226$

Metabolic Disease

$6.86 \mathrm{E}-06-2.68 \mathrm{E}-0269$

Gastrointestinal Disease

$1.82 \mathrm{E}-04-2.21 \mathrm{E}-0269$

Hepatic System Disease

$1.82 \mathrm{E}-04-2.21 \mathrm{E}-0254$

\section{Molecular and Cellular Functions}

Name

p-value

\#

Gene Expression

1.73E-05 - 8.05E-03 123

Cell Death and Survival

5.67E-05 - 3.00E-02 189

Cellular Growth and Proliferation

$2.01 \mathrm{E}-04-2.96 \mathrm{E}-02173$

Cellular Development

2.51E-04 - 2.80E-02 119

Lipid Metabolism

3.59E-04 - 2.57E-02 83

\section{Physiological System Development and Function}

Name

p-value

Organismal Development

3.52E-05 - 2.80E-02 102

Tissue Morphology

1.33E-04 - 2.77E-02 98

Organismal Survival

$1.71 \mathrm{E}-04-2.52 \mathrm{E}-02148$

Endocrine System Development and Function

2.28E-04 - 1.29E-02 35

Connective Tissue Development and Function

$2.51 \mathrm{E}-04-2.76 \mathrm{E}-0268$

(c) 2000-2014 Ingenuity Systems, Inc. All rights reserved. 


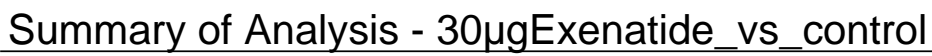

Top Canonical Pathways

Name

p-value

Ratio

Ketogenesis

3.36E-04

$4 / 21$

NRF2-mediated Oxidative Stress Response

6.24E-04 16/195

Aldosterone Signaling in Epithelial Cells

1.12E-03

$(0.082)$

Ketolysis

2.51E-03

$14 / 168$

2.62E-03

$3 / 19$

(0.158)

Insulin Receptor Signaling

$12 / 149$

(0.081)

Top Molecules

\section{Fold Change up-regulated}

\begin{tabular}{l} 
Molecules \\
\hline ZBTB16 \\
UPP2 \\
HMGCS2 \\
NPTX2 \\
NR1D1 \\
FKBP5
\end{tabular}




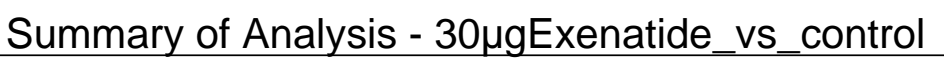

Acot1

$\uparrow 4.658$

NR1D2

$\uparrow 4.201$

USP2

$\uparrow 2.974$

MT1H

$\uparrow 2.608$

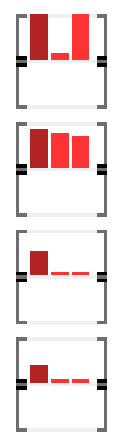

\section{Fold Change down-regulated}

\begin{tabular}{|c|c|c|}
\hline Molecules & Exp. Value & $\begin{array}{l}\text { Exp. } \\
\text { Char }\end{array}$ \\
\hline LDLR & $\downarrow-3.851$ & \\
\hline ATAD5 & $\downarrow-2.904$ & \\
\hline Akr1c14 & $\downarrow-2.578$ & \\
\hline PDE4B & $\downarrow-2.511$ & \\
\hline GAS7 & $\downarrow-2.451$ & \\
\hline FGF14 & $\downarrow-2.408$ & \\
\hline SGK1 & $\downarrow-2.313$ & \\
\hline RAB3IL1 & $\downarrow-2.199$ & \\
\hline PEG3 & $\downarrow-2.144$ & \\
\hline $\mathrm{CHIC1}$ & $\downarrow-2.066$ & \\
\hline
\end{tabular}


Top Upstream Regulators

Upstream Regulator

\section{D-glucose}

FOXO3

ARID2

PTEN

ATP7B p-value of overlap Predicted Activation

1.11E-05 2.54E-05

4.96E-05

$6.26 \mathrm{E}-05$

7.20E-05 
Top My Lists

Name

p-value

Ratio

My List angiogenesis 769

Top My Pathways

Name

p-value

Ratio

\section{Top Tox Lists}

Name

Liver Proliferation

p-value

Ratio

NRF2-mediated Oxidative Stress Response

3.07E-03 16/207

(0.077)

Nongenotoxic Hepatocarcinogenicity Biomarker Panel

4.96E-03

$16 / 234$

5.76E-03

(0.068)

Liver Necrosis/Cell Death

1.31E-02

PXR/RXR Activation

1.75E-02

(0.182)

$17 / 262$

(0.065)

$6 / 67$

(0.09) 


\section{Top Tox Functions}

\section{Assays: Clinical Chemistry and Hematology}

Name

p-value

\#

Increased Levels of Red Blood Cells

6.77E-03 - 6.77E-03 9

Increased Levels of Alkaline Phosphatase

3.84E-02 - 3.84E-02 6

Increased Levels of Hematocrit

$2.45 \mathrm{E}-01-2.45 \mathrm{E}-015$

Decreased Levels of Albumin

$2.90 \mathrm{E}-01-2.90 \mathrm{E}-011$

Increased Levels of AST

3.66E-01 - 3.66E-01 1

\section{Cardiotoxicity}

Name

p-value

3.73E-02 - 1.00E00 6

Cardiac Arrythmia

3.73E-02 - 5.83E-01 8

Cardiac Fibrosis

3.73E-02 - 4.55E-01 4

Cardiac Inflammation

3.73E-02 - 5.67E-01 14

Cardiac Necrosis/Cell Death

5.57E-02 - 6.14E-01 17

\section{Hepatotoxicity}

Name

p-value

Liver Steatosis

1.82E-04 - 5.84E-02 22

Liver Proliferation

1.45E-03 - 4.17E-01 16

Hepatocellular Carcinoma

5.07E-03 - 2.35E-01 23

Liver Hyperplasia/Hyperproliferation

5.07E-03 - 4.76E-01 29

Liver Inflammation/Hepatitis

1.01E-02 - 1.77E-01 17 


\section{Nephrotoxicity}

Name

Renal Atrophy

Renal Damage

Renal Inflammation

Renal Nephritis

Renal Tubule Injury p-value

1.10E-03 - 1.10E-03 5

3.73E-02 - 4.88E-01 9

3.73E-02 - 5.99E-01 11

3.73E-02 - 5.99E-01 11

3.73E-02 - 3.40E-01 5 